\title{
Clinical, bacteriological and immunological follow-up of household contacts of leprosy patients from a post-elimination area - Antioquia, Colombia
}

\author{
Nora Cardona-Castro/ ${ }^{+}$, Juan Camilo Beltrán-Alzate, Marcela Romero-Montoya \\ Instituto Colombiano de Medicina Tropical, Universidad CES, Cra 43A 52Sur-99, Sabaneta, Antioquia, Colombia
}

Follow-up of the household contacts (HHC) of leprosy patients is still the best strategy for early detection of leprosy. HHC from a post-elimination region of Colombia studied in 2001-2002 were re-contacted in 2007. They were tested at both times by clinical examination, bacillary index (BI), PCR from a slit skin smear (SSS) and anti PGL-1 IgM titres. Thirty-two of 61 HHC (52\%) were re-contacted. Nine HHC (28\%) showed sero-conversion and one had a skin lesion (BI negative, nested PCR positive). Periodic evaluation of HHC can contribute to the detection of infected $H H C$ as well as new and early leprosy cases.

Key words: household contacts - leprosy patients - follow-up - post-elimination region

Leprosy in Colombia is not a public health problem and control of leprosy is in the "post-elimination phase" (WHO 2006). The prevalence of leprosy in Colombia reported in 2008 was 0.87 per 100,000 habitants (SI-VIGILA 2008). Several regions of Colombia, however, show large differences in leprosy prevalence. For example, leprosy prevalence in state of Antioquia has fluctuated between 0.4-0.6 per 100,000 habitants, but there are regions in the states of Bolívar and Cesar where the prevalence ranges from 3.7 per 100,000 (SIVIGILA 2008). There were 345 new leprosy cases detected in Colombia in 2008 (SIVIGILA 2008); 3.52\% of these were in children under 15 years of age and the percentage of grade II disability among new cases was $7.79 \%$ (WHO 2006). The number of new cases has remained around 350-500 since the multi-drug therapy (MDT) was introduced in 1985 (SIVIGILA 2008), indicating that the therapy has not been effective in preventing transmission (Meima et al. 2004) and that the number of new cases remains the same in a region that is considered to be in a post-elimination phase. Household contacts (HHC) are a high-risk population for developing leprosy (Goulart et al. 2008). At present, the Leprosy Control Programs do not routinely use to detect clinical leprosy follow-up in HHC since only one clinical examination is stipulated as a control measure at the time the index case is diagnosed. Nevertheless, clinical examination is not a good tool for detecting subclinical leprosy due to the fact that leprosy is a disease with a slow onset and the symptoms are difficult to detect in

Financial support: Dirección Seccional de Salud de Antioquia, Instituto Colombiano de Medicina Tropical - Universidad CES

+Corresponding author: ncardona@ces.edu.co

Received 27 April 2009

Accepted 26 August 2009 the early stage of infection (Lockwood 2002). Therefore, diagnosis is often delayed, contributing to the onset of the disease and allowing transmission and sequelae (Lockwood \& Kumar 2004). The use of other tests in addition to periodic evaluations by clinical exam to detect infected HHC, as well as detailing their immune response and bacteriological state, can contribute to the identification of infected HHC and new leprosy cases (Cardona-Castro et al. 2005).

The first study in 2001-2002 included $61 \mathrm{HHC}$ and the follow-up in 2007 was able to re-contact $32 \mathrm{HHC}$ (52.4\%). During the first evaluation, these $32 \mathrm{HHC}$ showed negative titres for anti PGL-1 IgM and neither clinical signs nor symptoms suspicious of leprosy were recorded. Twelve leprosy patients were the index cases of the $61 \mathrm{HHC}$; eight were male and four were female. These index cases included 11 patients with diagnosis of paucibacillary $(\mathrm{PB})$ leprosy [bacillary index $(\mathrm{BI})=0$ ] and one patient with multibacillary (MB) leprosy (BI = $2,4)$ at the time of the first evaluation. All of the index cases were re-contacted in 2007.

HHC and index cases were examined for signs or symptoms of skin and peripheral nervous leprosy. HHC and patients were tested for BI using the Ziehl Nielsen stain. Slit skin smear (SSS) from ear lobes, elbows and lesions were taken, along with nasal mucus samples (NS). The smears were examined for the presence of acid-fast bacilli (AFB). BI was recorded (Isenberg 1992). M. leprae DNA from SSS and NS of HHC was extracted using the DNeasy $\mathrm{kit}^{\mathbb{\circledR}}$. Nested PCR was carried out using the primers LP1-LP2 and LP3-LP4 (Donoghue et al. 2001). HHC serum samples were tested for anti PGL-1 IgM, which was applied using the native PGL-1 antigen provided by Dr. Patrick Brennan from Colorado State University, USA, following a previously described methodology (Cho et al. 1983). After explanation of the study and procedures, volunteers provided informed consent. In agreement with resolution 008430 of 1993 that regulates health studies in Colombia, this investigation was considered to involve minimal risk. 
The ages of the HHC ranged from 11-79 years old with an average age of 41.5 years. Nine of the HHC were males and 23 were females. Nine HHC who had negative titres at the time of the first study showed positive titres for anti PGL-1 IgM in 2007 (sero-conversion of $28 \%$ ); one of them was an 11-year-old girl. One HHC had one light, hypo-pigmented skin lesion $(2 \times 3 \mathrm{~cm})$, located on her left thigh with an anaesthetic area surrounding the lesion, which was $\mathrm{BI}$ negative and nested PCR positive from her SSS. This HHC was diagnosed as a PB patient and received the appropriate treatment, according to WHO recommendations. None of the HHC showed positive BI, including the new patient. For the nested PCR, only the new patient was positive. The 12 index cases of the HHC had a BI of zero and the clinical exam did not reveal active lesions of leprosy and/or clinical relapse in the second evaluation. Grade I and II disability was observed in $100 \%$ of patients.

Periodic evaluation of HHC by clinical exam, immune response and bacteriological state can contribute to the detection of infected HHC for new and early leprosy cases. Due to migration, not all of the participants could be followed, resulting in a loss of information. Only $32(52.5 \%)$ of the study's first round participants were followed up. We are now recommending a yearly follow-up of $\mathrm{HHC}$ in an effort to reduce the risk of losing critical information. Nine of $32 \mathrm{HHC}$ showed negative titres for PGL-1 during the first study, but after six years they now have positive titres (a $28 \%$ sero-conversion rate), which should signify either new exposition or infection or a slow immune response after contact with $M$. leprae. It is important to note that one of the HHC who sero-converted was an 11-year-old girl, which demonstrates the importance of follow-up of M. leprae infection in children for early disease detection and disability prevention in a young population. Other studies have shown frequencies of infected contacts that range from 13-93\% (Meeker et al. 1986, Amezcua et al. 1987, Cardona-Castro et al. 2005, 2008). Early detection of PB leprosy was possible in one $\mathrm{HHC}$ who had a unique lesion and the diagnosis was confirmed by positive nested PCR. Treatment for PB leprosy was initiated in accordance with WHO recommendations. Results confirm that the use of multiple testing modalities and follow-up for $\mathrm{HHC}$ is important because the clinical exam done at the time of detection of the index case is not sufficient to know whether HHC are infected.

The 29 HHC that were not included in the followup were negative for anti PGL-1 IgM titres in the first study (2001-2002). The results of the evolution of these $29 \mathrm{HHC}$ could change the results of this follow-up, a fact that represents a bias of this study.
Results reveal that leprosy transmission is occurring in post-elimination areas. The possible failure of treatment due to non-adherence or the appearance of antibiotic resistance need to be evaluated (Hernández et al. 2008). Classification as a post-elimination area contributes to the lack of financial resources for surveillance and leprosy public health control measures in a high-risk population.

\section{REFERENCES}

Amezcua ME, Escobar-Gutierrez A, Mayen E, Cazares JV 1987. Sensitivity and specificity of the FLA-ABS test for leprosy in Mexican populations. Int J Lepr Other Mycobact Dis 55: 286-292.

Cardona-Castro N, Beltrán C, Manrique R 2008. Survey to identify Mycobacterium leprae - infected household contacts of patients from prevalent regions of leprosy in Colombia. Mem Inst Oswaldo Cruz 103: 332-336.

Cardona-Castro N, Restrepo-Jaramillo S, Gil de la Ossa M, Brennan P 2005. Infection by Mycobacterium leprae of household contacts of lepromatous leprosy patients from a post-elimination leprosy region of Colombia. Mem Inst Oswaldo Cruz 100: 703-707.

Cho SN, Yanagihara DL, Hunter SW, Gelber RH, Brennan PJ 1983. Serological specificity of phenolic glycolipid I from Mycobacterium leprae and use in serodiagnosis of leprosy. Infect Immun 41: 1077-1083.

Donoghue HD, Holton J, Spielgelman M 2001. PCR primers that can detect low levels of Mycobacterium leprae DNA. J Med Microbiol 50: 177-182.

Goulart IM, Bernardes Souza DO, Marques CR, Pimenta VL, Gonçalves MA, Goulart LR 2008. Risk and protective factors for leprosy development determined by epidemiological surveillance of household contacts. Clin Vaccine Immunol 15: 101-105.

Hernández E, Cardona-Castro N, Rodríguez G, Villegas S, Beltrán C, Kimura M, Vissa V, Gómez Y 2008. Estudio de resistencia a la rifampicina y la dapsona en tres pacientes con recurrencia de lepra. Rev Panam Salud Publica 23: 73-77.

Isenberg H 1992. Clinical microbiology procedures handbook, American Society for Microbiology, Washington, p. 7.2.2-7.2.4.

Lockwood DN 2002. Leprosy elimination - a virtual phenomenon or a reality? BMJ 324: 1516-1518.

Lockwood DN, Kumar B 2004.Treatment of leprosy. BMJ 328: 1447-1448.

Meeker HC, Levis WR, Sersen E, Schuller-Levis G, Brennan PJ, Buchanan TM 1986. ELISA detection of IgM antibodies against phenolic glycolipid-I in the management of leprosy: a comparison between laboratories. Int J Lepr Other Mycobact Dis 54: 530-539.

Meima A, Smith W, van Ootmarssen G, Richardus JH, Habbema DF 2004. The future incidence of leprosy: a scenario analysis. Bull World Health Org 82: 373-380.

SIVIGILA 2008. Situación de la lepra en Colombia. Available from: www.minsalud.gov.co.

WHO - World Health Organization 2006. Global strategy for further reducing the leprosy burden and sustaining leprosy control activities 2006-2010. Operational guidelines. Lepr Rev 77: 1-50. 